\title{
Astronomy for African development
}

\author{
Kevindran Govender \\ South African Astronomical Observatory, \\ P.O. Box 9, Observatory, Cape Town, 7935 South Africa \\ email: kg@saao.ac.za
}

\begin{abstract}
In recent years there have been a number of efforts across Africa to develop the field of astronomy as well as to reap benefit from astronomy for African people. This presentation will discuss the case of the SALT (Southern African Large Telescope) Collateral Benefits Programme (SCBP) which was set up to ensure societal benefit from astronomy. With African society as the target, the SCBP has embarked on various projects from school level education to public understanding of science to socio-economic development, the latter mainly being felt in the rural communities surrounding the South African Astronomical Observatory (home to SALT). A development plan for "Astronomy in Africa" will also be discussed. This plan has been drawn up with input from all over Africa and themed "Astronomy for Education". The Africa case stands as a good example for the IYA cornerstone project "Developing Astronomy Globally" which focuses on developing regions.
\end{abstract}

Keywords. development, SALT, Africa, International Year of Astronomy

\section{Introduction}

Astronomy has always captured the imagination of human beings and still plays a rôle in almost every culture on Earth. However, in the modern era where the issue of 'development' is foremost on people's minds, one has to look carefully at tangible benefits in the context of challenges facing people today. The millennium development goals serve as an idea of the world's major challenges and they highlight issues such as poverty. Most people affected by poverty would find it difficult to accept that large amounts of resources are spent on astronomy while their families starve. Although there are justifiable reasons, on a higher political level, as to why this situation exists, it is more difficult to obtain the blessings of all members of society.

The Southern African region has always played a significant role in terms of astronomy on the African continent, with South Africa as the regional strong point. The three major observatories in Southern Africa are: the South African Astronomical Observatory (SAAO) which is home to the Southern African Large Telescope (SALT); the High Energy Stereoscopic System (HESS) in Namibia; and the Karoo Array Telescope (MeerKAT) also in South Africa. In the South African context the government's investment in astronomy has often been controversial though never significantly opposed. Being a country with a number of socio-economic and developmental challenges following a difficult history, South Africa has embarked on a visionary journey in recognition of the importance of astronomy for development. In general South Africa's attitude towards science and technology has been exemplary and is clearly reflected in national policies such as the White Paper on Science and Technology (1996)†. An extract from this paper captures the vision behind the investment in 'big science' projects:

"The well-being of scientific activity in South Africa is intimately linked to material factors. World-wide there is a clear trend for curiosity-driven research to

$\dagger$ available at www.dac.gov.za/white_paper.htm 
increase as a function of national per capita income. Nevertheless, there is a danger of adopting too economistic a viewpoint. Even at our current stage of development, there is a need to recognise the importance of the knowledge-generating function of research, particularly in the higher education sector. Human wonder and curiosity and the ability to recognise serendipitous discovery account for much of scientific progress. Basic enquiry, as opposed to a formula-driven approach, is absolutely essential, particularly at the universities and technikons, which deal with young minds. It is important that fundamental research activity not be regarded as impractical, because it is the preserver of standards without which, in the long term, the applied sciences will also die.

Scientific endeavour is not purely utilitarian in its objectives and has important associated cultural and social values. It is also important to maintain a basic competence in "flagship" sciences such as physics and astronomy for cultural reasons. Not to offer them would be to take a negative view of our future - the view that we are a second class nation, chained forever to the treadmill of feeding and clothing ourselves."

South Africa has also recently brought into law the Astronomy Geographic Advantage Act "to provide for the preservation and protection of areas within the Republic that are uniquely suited for optical and radio astronomy; to provide for intergovernmental cooperation and public consultation on matters concerning nationally significant astronomy advantage areas; and to provide for matters connected therewith." $\dagger$

With such visions for astronomy at a high governmental level in South Africa, the SALT Collateral Benefits Programme (SCBP) was set up within the SAAO to ensure tangible benefit from astronomy to African society. As such this programme serves as an ideal case study when examining the impact of astronomy for African development.

\section{Overview of astronomy for African development}

Through various discussions with rôle players across Africa, there arose 5 areas of development in which astronomy can play a key role: Education; Research; Public understanding of Science; Partnerships; and Job Creation (specifically in the field of tourism or 'astro-tourism').

\subsection{Education}

In keeping with the spirit of the New Partnership for Africa's Development (NEPAD), which identifies education as a priority, astronomy, through its innate ability to stimulate curiosity, can be used as a rallying point for the development of a strong learning culture. This is with the view that education, which is probably the most sustainable form of development, remains one of the most important and crucial challenges facing the African continent, a challenge which could be the single most significant barrier to peace and development. In Africa, astronomy remains one of the most accessible of all sciences, with the biggest laboratory (a dark night sky) being most available to people in rural areas. Night-time satellite images of the earth clearly illustrate this. The widespread buy-in by the education and outreach sectors in South Africa has proven how powerful the impact of astronomy can be on education.

\subsection{Research}

The need for the development of a strong research community is clear from policies and structures already in place within the African Union, more specifically NEPAD

\footnotetext{
$\dagger$ www.info.gov.za/view/DownloadFileAction?id=83962

$\ddagger$ see www.nepad.org
} 
not to mention the policies of individual countries such as South Africa who aim to invest over $1 \%$ of their Gross Domestic Product (GDP) in science and technology. In such an environment astronomy can stimulate research in many other science fields. In South Africa for example the development of large telescopes have had an impact on technological capabilities begging the need for high level optical, construction and data manipulation expertise. The mathematics and theoretical physics communities tend also to overlap or interact closely with the astronomy community. In fact the pre-requisite for following a career in astronomy is a solid mathematics or physics background. The development of astronomy thus leads inevitably to the development of those respective university research departments.

\subsection{Public Understanding of Science}

In many developing countries one finds that modern scientific knowledge amongst the public is not very strong. In many cases traditional knowledge or superstitions dominate, leading to many misconceptions. Astronomy plays a key rôle in addressing this often sensitive relationship between traditional and scientific knowledge systems since the sky is something that virtually every culture is already familiar with (in one way or the other). Indigenous astronomy is usually a very interesting topic of conversation and an easy way to start a gradual process of introducing people to the modern understanding of the universe. For example, a conversation about beliefs surrounding an eclipse followed by a practical demonstration of the principle has been found to be very effective in stimulating scientific thinking. By bringing to the public the things we know about the universe and more importantly, how we know them (technologies employed, scientific method, etc), we stimulate a public understanding and appreciation of science and technology.

One must stress though that the conversation certainly goes both ways, and an acknowledgement of indigenous knowledge is important and essential in building the relationship with and pride of a people. Indigenous astronomical knowledge in Africa (such as the asterism isiLimela, aka Pleiades, which indicated the start of the planting season) serves as proof of the advanced thinking and observations of our ancestorst. In fact, archaeological finds in Timbuktu and other parts of Africa strongly suggest that Africans were doing astronomy hundreds if not thousands of years ago. Such discussions support the fact that the field of astronomy does in fact belong to us all as human beings, and is certainly not something exclusive that can only be enjoyed by the wealthy or privileged.

\subsection{Partnerships}

The 8th United Nations Millennium Development Goal is to 'Develop a Global Partnership for Development' $\ddagger$. The need for partnerships are also highlighted in the sentiments and spirit of the African Union and regional co-operation bodies such as the Southern African Development Community (SADC), the East African Community (EAC), and the Economic Community for West African States (ECOWAS). By its very nature astronomy stimulates a need to form partnerships with other countries, not just for the large financial investments but also for the geographic distribution of observatories. The process of developing astronomy would speak directly to and support a number of bilateral and multilateral agreements already in place across Africa.

\subsection{Job Creation (astro-tourism)}

With appropriate marketing and media coverage the field of astronomy in South Africa is growing into a significant attraction for tourists. The number of tourists to the small town

$\dagger$ www.saao.ac.za/public-info/sun-moon-stars/african-starlore

$\ddagger$ www.unmillenniumproject.org/goals/index.htm 
of Sutherland (home to the SAAO and SALT) has become so significant that a number of small businesses have sprung up all around the town, ranging from accommodation to restaurants to souvenir shops. The demand for tours at SALT during certain times of the year far exceeds the capacity of the observatory which has resulted in the training and involvement of unemployed individuals from Sutherland. As the lights of cities continue to grow, Africa has been holding on to one of its greatest untapped resources: a dark and clear night sky. A growing number of commercial African tourism companies are making the most of this valuable resource using 'amateur' telescopes and attracting business from foreign visitors as well as corporate companies. In Africa there is great potential for 'astro-tourism' to be developed just as much as 'eco-tourism' already has been. The International Year of Astronomy, in bringing attention to the field, is the opportune time to explore this potential further.

\section{SALT collateral benefits programme (SCBP)}

\subsection{Original $S C B$}

In 1998, the South African government approved the construction of SALT in Sutherland, Northern Cape Province. The government agreed to contribute the major part of the capital construction costs with the remaining costs sourced from international partners. SALT would enable South Africa to remain internationally competitiv e in astronomy well into the 21st century.

The original SALT Collateral Benefits Programme was established during the construction of SALT and its objectives were directed at the benefits derived by society from building this large telescope. It was designed to maximise benefits from the investment of public funds through five main thrusts: SALT industrial empowerment; SALT educational empowerment; Public outreach and direct educational benefits; science education visitor centres; and SALT as an African facility.

The overarching goal was always to ensure that the maximum collateral benefits are derived from the SALT project to advance the economy, technology, and society of Africa.

\subsection{Current $S C B P$}

In January 2006, with the SCBP division under new leadership and with SALT moving from the construction to operational phase, the rôle and purpose of the SCBP was reevaluated. The new strategy was based on a number of documents including the business plans of the original SCBP, the National Research Foundation (NRF) and the South African Agency for Science and Technology Advancement (SAASTA). Recommendations made from external evaluations of the SCBP, together with input from the original champions of the programme, were also taken into account.

From the outset, the programme always aimed to be seen as being owned by the staff of the whole of the SAAO (and SALT) and managed by the Collateral Benefits Division. Thus one should not view the SCBP in isolation from the rest of the SAAO but rather a common ground which can be utilised by all staff that may have ideas or skills to contribute towards a common vision. An additional benefit of this programme would be in terms of its use at other scientific institutions. If proven to be successful, this strategy was envisaged to serve as a model that could readily be implemented at any science research based institution in order to derive maximum benefit for society from their respective scientific activities.

Although the main focus of activities would be in the immediate vicinity of the SAAO sites (Karoo-Hoogland and Cape Town Metropolitan) the programme would seek to make an impact across the continent. The vision of the SCBP aligned directly to that of the 
parent organization, the NRF: A prosperous South Africa and African continent steeped in a knowledge culture, free of widespread diseases and poverty, and proud contributors to the well-being of humanity.

The SCBP would focus on 3 areas: education in mathematics, science, engineering and technology to supply the country and the continent with well trained and motivated professionals in substantially increased numbers; science communication and awareness to effectively engage with the public in order to disseminate relevant information in the fields of astronomy and space science; and socio-economic development in order to contribute to a better quality of life for all people, especially the disadvantaged.

\subsubsection{Education in Mathematics, Science, Engineering and Technology}

The biggest impact that SALT and astronomy has had on the people in South Africa has probably been in education. SALT is an icon that is known by virtually every school learner in the country: it is even a part of the school curriculum at various levels. What it means to a young South African is that there are great opportunities in their own country in the fields of science, engineering and technology. The SCBP uses this icon to inspire learners towards careers in these fields. Astronomy and SALT are also used as a tool for teaching concepts in mathematics, science and technology at school level. A brief description of a selection of educational activities is given below.

- Educator and learner workshops: The SCBP conducts workshops for both educators and learners on a regular basis. These vary from building telescopes and spectroscopes to a variety of hands on activities that help one to understand astronomical concepts such as seasons, eclipses, etc.

- Work shadow: Due to the scarcity of high level skills in South Africa and the need to expose young people to different careers, the work shadow programme links learners to technical staff and astronomers who they can 'shadow' for a few days to learn about the respective career.

- Science Clubs: The SCBP coordinates the formation and networking of science clubs across the country. Although the focus is usually on astronomy all science clubs are part of the network.

- Resource Development: New educational resources are constantly developed or adapted from existing ones. These resources are shared with all collaborators nationally and internationally.

- Outreach: Telescopes are taken out to schools and science clubs who cannot travel to the observatory. Although it is usually for evening talks and stargazing, daytime activities are also possible using telescopes with solar filters.

- Universe Awareness (UNAWE): The SCBP first became involved in the UNAWE programme (one of the 11 cornerstones for IYA2009) in 2006. UNAWE activities use astronomy as a tool for inspiration and are targeted at very young children especially in rural areas.

- Development of the Sutherland Schools: The SCBP is closely involved in efforts to develop and enhance the schooling in the town of Sutherland. This comes in the form of various projects in partnership with the Department of Education as well as direct interventions such as extra lessons and educator development.

- Scholarships: The SCBP coordinates school level and university level scholarships awarded to deserving students in the fields of astronomy and physics. The school level scholarship serves as encouragement to follow a science career. The SALT international partner universities also offer scholarships for South African students to pursue postgraduate studies overseas. 


\subsubsection{Science communication and awareness}

Public understanding of science and technology, especially in the rural Northern Cape area where SALT is located, is generally very low. The SCBP organizes events and activities that enable greater conversation between the observatory and the community. Star-gazing events in the town are accompanied by talks by astronomers/engineers. Open discussions are held about the community's belief systems as well as the science being done at the observatory. Free SALT tours are also offered (transport provided at no cost) for people in Sutherland. Examples of some public outreach activities in Sutherland and across South Africa are listed below.

- Tour programme at Sutherland: Both day and night tours are coordinated by the $\mathrm{SCBP}$ to the research telescopes and visitor centre in Sutherland. Two visitor telescopes (14 and 16 inch reflectors) are available for stargazing.

- Public Visits in Cape Town: Public visits to the observatory in Cape Town are either in the form of open nights, held monthly or ad hoc day visits arranged through the SCBP.

- Development of resources for public: Materials such as information flyers and posters as well as astronomy related memorabilia are developed for the public.

- Distribution of resources to various expos and festivals: Due to the number of events across the country it is not always possible to send staff to all of them. SCBP therefore coordinates with partners across the country to have astronomy represented at such events and provides the necessary resources.

- Media and Press releases: Regular release of interesting astronomy related news to the press. These include anything from recent research being performed to astronomical events like eclipses.

- Indigenous Astronomy: There are constantly projects underway with partners in other institutions regarding the investigation and development of resources concerning indigenous astronomy.

- Visitor Centre Developments: Exhibits at the visitor centre in Sutherland are constantly under development illustrating different concepts of astronomy in a user friendly manner.

- Monthly "What's up" resources: Online and print explanations of what can be seen in the sky during that particular month.

\subsubsection{Socio-economic development}

This focus area, although probably the most important to people in poorer communities, is also the most difficult to directly address. The SCBP is constantly involved in discussions with local communities on activities and programmes that will help to uplift the socio-economic status of the area. The difficulty comes in drawing the line between what we can do from the observatory's side and what the local municipality is mandated to do by the national government. The biggest impact on the community since the inauguration of SALT has certainly been in the field of tourism. In the first year after the SALT inauguration the number of visitors to the small town of Sutherland jumped up from a few hundred to over 13,000 visitors per annum. This has resulted in a rapid increase in the number of guest houses, coffee shops and tourism related businesses such as the Arts \& Crafts project for the unemployed. SALT has been involved in a number of socio-economic development projects on various levels, from being a partner in funding proposals to actual implementation on the ground. Some of the highlights are listed below.

- Close working relationships with various development stakeholders: Partnerships have been established to enhance the development in the region. Partners include: 
Karoo-Hoogland Municipality; National Development Agency; Tourism Enterprise Programme; Succulent Karoo Ecosystem Programme; Military Service Corps

- Regular community meetings hosted by SCBP: These are basically brainstorming sessions where the community has an opportunity to engage with the observatory and come up with ways in which the people of the area can benefit from the observatory being there.

- Exploration of the various socio-economic roles of SALT in South African Society: There is an ongoing process of engagement with government both locally and nationally to explore ways in which greater benefits can be reaped from astronomy in general and SALT in particular.

- Community Tour guides: Unemployed individuals from the Sutherland community were selected and trained to conduct basic tours of the facilities. This opportunity generally increases the marketability of the individuals and many have found jobs soon after. Thus the project is ongoing and more guides are brought in as some of them leave.

- Tarring of roads: There are ongoing negotiations which the observatory are involved in, together with various stakeholders, to improve the quality of the roads in the area. Better roads would imply greater accessibility to the observatory and thus greater tourism for the town. The impact of better roads reaches schools and local businesses in the area.

- Sourcing funding for development: The SCBP has been involved in various partnerships, especially with the local municipality to source funding for development projects that would lead to job creation. The significant political status of SALT helps in the process of fundraising.

- Arts and Crafts programme: The tourism generated by SALT has led to initiatives such as the Arts and Crafts programme which empowers individuals from the community around SALT to develop marketable products for tourists. The SCBP runs a small kiosk at its visitor centre which markets these products.

\subsection{SCBP and the International Year of Astronomy 2009 (IYA2009)}

In December 2007 the United Nations General Assembly declared that 2009 would be the International Year of Astronomy (IYA2009). This is a global celebration of astronomy and its contribution to society and culture, with a strong emphasis on education, public engagement and the involvement of young people. Events will take place at national, regional, and global levels throughout the whole of 2009 .

The SCBP has seen this year as an opportunity to enhance the impact of astronomy in society and has taken a lead rôle in the celebrations of IYA2009 across South Africa and Africa. Various activities have been undertaken from the consolidation of resources to the rallying together of stakeholders in order to make IYA2009 a success. Highlights of some of these activities are included below:

- Astronomy outreach stakeholder meetings: Participants included observatories, planetariums, amateur astronomy groups, science centres and government departments.

- Survey of astronomy education and outreach: Soon after the initial stakeholder meetings an open survey was carried out to establish who was doing what across the country, and to seek input as to where astronomy education and outreach in South Africa should be heading.

- AstroNet email list: As the number of stakeholders increased the AstroNet email list was set up as a means of open communication amongst all concerned parties dealing with astronomy education and outreach. This email list has since grown dramatically to include people from all over Africa and the rest of the world and is now an invaluable resource for news and events in the field. 
- Website astronomy2009.org.za : The coordination was leaning strongly towards IYA 2009 and thus the website that was set up served two purposes, consolidation of existing South African efforts as well as the coordination of activities for IYA2009. A section of this website was specifically set aside for the coordination of IYA2009 in Africa.

- Consolidation of astronomical resources: With a growing number of individuals and organisations across the country producing resources there was a glaring need for a consolidation of materials. Calls were sent for astronomy education and outreach materials and these were consolidated and categorised onto a common database, freely accessible online.

- AstroGuide: As the number of people wanting to get involved in astronomy outreach increased, a peer reviewed resource was produced to provide guidance for any interested parties. The 'AstroGuide' was a bulleted list of important points that one should cover when talking about astronomy. Its usefulness was hailed by the community and its popularity grew, especially amongst other African countries. This peer review process using the AstroNet list and expertise at the observatories serves as an example for a peer review mechanism to be put in place for other astronomy resources.

- AstroCD: In recognition of the fact that many schools and organisations around Africa have access to computers but not necessarily the internet, a CD full of free electronic resources was put together. This CD contained local and international educational resources as well as free software used in the teaching of astronomy. Popularity of the AstroCD soared amongst schools and astronomy stakeholders and hundreds of CDs to date have been distributed free of charge.

- "Essential Astronomy" Outreach Training Workshops: In preparation for IYA2009 and to ensure greater reach of astronomy, a number of workshops were held across the country targeting education and outreach staff who dealt with educators, learners and the public on a regular basis. The idea was that the instructor for any general science education or outreach programme should be equipped with the tools and knowhow to use astronomy in their activities. This workshop proved incredibly popular and has since reached over 200 individuals from across South Africa and a number of other African countries including Kenya, Namibia, Nigeria, Uganda, Zimbabwe, Mozambique and Lesotho.

- Securing of funds: The Department of Science and Technology† had to be lobbied for IYA2009 funding, a process that began long before 2009. The dissemination of the funds was coordinated by the South African Agency for Science and Technology Advancement (SAASTA) with strong support from the SCBP.

Due to its strength on the African continent, South Africa (led by the SCBP), has also been supporting IYA2009 activities in various countries across Africa.

\section{A plan for Africa}

The plan for "Astronomy Education and Outreach in Africa" was built on input from a number of people across Africa (and beyond) who are involved in astronomy, space science or general science education in some way. This plan was developed in anticipation of IYA2009 with the intention of lasting well beyond 2009. The need was influenced by the glaring lack of representation by African countries on the IYA2009 and astronomy scenes. In the build up towards IYA2009 the developers of this plan wanted to ensure that Africa had a voice and reaped as much benefit as possible from this global event.

$\dagger$ www.dst.gov.za 
Since then a task group has been formed to take this plan forward. In building up the astronomy community in Africa, the task group aims to use the subject to spark an interest not only in science, engineering and mathematics disciplines, but also in education in general. Thus IYA2009 would be used as a launching pad for a network of African individuals and organizations who intend to work together into the future using astronomy to enhance education in Africa. With this in mind the whole plan has been themed "Astronomy for Education".

The vision for the plan was specifically designed to address concerns in Africa. It thus reads "The continent of Africa, with an ever-growing astronomy research community, united in the fields of education and outreach, working together and sharing resources, such that the people of Africa are educated, especially in the fields of science, engineering and technology."

The vision will be realised through four core areas, with a focus on building and supporting human resources:

- Enhance the teaching and interest in Mathematics and Science in schools

- Enhance the teaching and research interest in astronomy in universities

- Increase the awareness and knowledge of science amongst the public

- Support and encourage an African network

The plan recommends that the pan-African network remain an online and dynamic structure keeping informed and in contact through email and a website for Africa. Within each country however, is required a driver (a single point of contact) along with a team that comprises the steering committee for that country. This structure is subject to change as more countries become more actively involved and provide input.

It is important to note the spirit with which this plan was constructed and the principles that guide it. Firstly it is about encouraging collaboration both nationally, within any particular country, and internationally, across Africa and beyond. It is critical to support and enhance rather than reinvent programmes. In this sense we certainly are looking to build on the shoulders of other astronomy related programmes. Vital to the effort will be to ensure adequate monitoring, evaluation and quality assurance, so that we can measure the success and shape the plan going forward. A conspicuous challenge in Africa is the lack of sufficient skills so the development and support of human resources is a pivotal principle. IYA2009 is just the start though and in order to have a lasting impact beyond 2009, we must ensure that projects are sustainable at every stage.

\section{Developing Astronomy globally}

'Developing Astronomy Globally' is an IYA2009 cornerstone project (one of 11) aimed at the various countries that do not have strong astronomy communities. The 3 different levels or focus areas of development identified for this project are: professional development which will address tertiary education, research training and research infrastructure in order to build professional astronomy research capacity within the country; development of the public understanding of astronomy which will target the general public and take the form of activities and events that stimulate an interest in astronomy, as well as the development of an amateur astronomy community and the development of astronomy communicators; and school-level education development which will address primary and secondary education.

There are two main goals of this cornerstone. Firstly it aims to use the momentum of IYA2009 to stimulate the establishment and enhancement of regional structures/networks around the world that would focus on the development of astronomy. These structures would support current and future development work of the IAU and other programmes. 
Secondly it aims to ensure sufficient reach and benefit of IYA2009 and other cornerstones to developing regions. Achieving these goals would result in an increase in the number of developing countries actively participating in IYA2009 as well as regional nodes in developing areas providing strong international support networks and collaborative projects that last beyond 2009 .

Implementation of this cornerstone will rely on strong collaborations with each of the other cornerstone projects as well as a close working relationship with the IAU Commission 46 and other similar bodies/programmes with common objectives. The implementation would follow a regional approach targeting 8 regions of the world, namely: North America; Latin America (including Central America and the Caribbean); Europe; Middle East and North Africa; Sub-Saharan Africa; Central Asia; Far East and South-East Asia; Oceania (including Australia and New Zealand).

An immediate deliverable of this cornerstone is a survey of the status of astronomy within various developing regions. This survey would map each of the three focus areas against their respective status for the country in question. The status would be classified according to four phases (phase 1 being most well developed and phase 4 being least developed). The idea is that, based on this mapping, certain applicable actions would be suggested or implemented in that country. All actions would ideally be coordinated by the regional structures in conjunction with the international office.

\section{Conclusion}

The significance and relevance of astronomy for African development has been demonstrated from programmes already implemented and underway. It is clear from the example and experiences of the SCBP that the field of astronomy does have the potential to impact greatly on society in ways that are comprehensible and palatable to all sectors of the population. Projects like the 'Astronomy for Education' plan for Africa and the 'Developing Astronomy Globally' cornerstone project are geared towards taking these benefits to much higher and far reaching levels.

\section{References}

Holbrook, J. C., Medupe, R. T. \& Urama, J. O. (eds) 2008, African Cultural Astronomy: Current Archaeoastronomy and Ethnoastronomy research in Africa (Berlin: Springer)

Martinez, P. 2007, in IAU Special Session 5, Astronomy for the Developing World, J. B. Hearnshaw and P. Martinez (eds) (Cambridge: Cambridge University Press), p. 89 